\title{
5-FU resistance abrogates the amplified cytotoxic effects induced by inhibiting checkpoint kinase 1 in p53-mutated colon cancer cells
}

\author{
TOMOFUMI AKASAKA ${ }^{1}$, MASAHIKO TSUJII ${ }^{1}$, JUMPEI KONDO ${ }^{1}$, YOSHITO HAYASHI $^{1}$, JIN YING $^{1}$, \\ YUQUAN LU ${ }^{2}$, MOTOHIKO KATO ${ }^{3}$, TAKUYA YAMADA ${ }^{4}$, SHUNSUKE YAMAMOTO ${ }^{5}$, TAKUYA INOUE ${ }^{1}$, \\ YOSHIKI TSUJII ${ }^{1}$, AKIRA MAEKAWA ${ }^{1}$, TETSUJI FUJINAGA ${ }^{1}$, ERI SHIRAISHI ${ }^{1}$, SATOSHI HIYAMA ${ }^{1}$, \\ TAKAHIRO INOUE ${ }^{1}$, SHINICHIRO SHINZAKI $^{1}$, KENJI WATABE $^{1}$, TSUTOMU NISHIDA $^{6}$, \\ HIDEKI IIJIMA $^{1}$ and TETSUO TAKEHARA ${ }^{1}$
}

\begin{abstract}
Departments of ${ }^{1}$ Gastroenterology and Hepatology and ${ }^{2}$ Environmental and Preventive Medicine, Osaka University Graduate School of Medicine, Suita, Osaka 565-0871; ${ }^{3}$ Division of Gastroenterology, National Hospital

Organization Tokyo Medical Center, Tokyo 152-8902; ${ }^{4}$ Department of Gastroenterology, Osaka National Hospital, Osaka 540-0006; ${ }^{5}$ Department of Gastroenterology, Osaka Rosai Hospital, Sakai, Osaka 591-8025;

${ }^{6}$ Department of Gastroenterology, Toyonaka Municipal Hospital, Toyonaka, Osaka 560-8565, Japan
\end{abstract}

Received July 16, 2014; Accepted September 3, 2014

DOI: 10.3892/ijo.2014.2693

\begin{abstract}
The emergence of chemoresistance is a major limitation of current cancer therapies, and checkpoint kinase (Chk1) 1 positively correlates with resistance to chemo- or radio-therapy. Cancer cells lacking p53 pathways are completely dependent on the $\mathrm{S}$ and $\mathrm{G} 2 / \mathrm{M}$ checkpoints via Chk1; therefore, Chk1 inhibition enhances the cytotoxicity of DNA-damaging agents only in p53-deficient cells. However, little is known about the synergistic effect of Chk1 inhibition with 5-FU, the most frequently used antimetabolite, in chemoresistant colorectal cells. In this study, we found that 5-FU induced S-phase arrest only in p53-deficient colorectal cancer cells. 5-FU treatment induced DNA damage and activation of ataxia telangiectasia mutated (ATM) and Chk1, leading to S-phase arrest, and Chk1 inhibition using SB218078 reduced $\mathrm{S}$-phase arrest and increased apoptosis in the presence of 5-FU. In contrast, in p53-deficient, 5-FU-resistant (5FUR) colon cancer cells that we developed, 5-FU enhanced DNA damage but did not induce Chk1/ATM activation or cell cycle arrest. SB218078 in combination with 5-FU did not induce apoptosis. These results indicate that 5-FU-resistance abrogated the anticancer effect amplified by Chk1 inhibition, even in p53-deficient cancer cells.
\end{abstract}

Correspondence to: Professor Tetsuo Takehara, Department of Gastroenterology and Hepatology, Osaka University Graduate School of Medicine, 2-2 Yamada-oka, Suita, Osaka 565-0871, Japan

E-mail: takehara@gh.med.osaka-u.ac.jp

Key words: Chk1, p53, 5-fluorouracil, colorectal cancer

\section{Introduction}

Many cancer therapies effectively kill proliferating tumor cells by causing DNA damage. However, a major limitation of current therapies is the emergence of resistance following the initial treatment. Various mechanisms, including, altered drug transport, increased expression of enzymes that the drugs target, enhanced catabolization of the drug by an enzyme, and/or increased tolerance of the cells to genotoxic stress via cell cycle checkpoints, DNA repair, and/or apoptosis, have been implicated in chemoresistance (1).

For the treatment of major solid tumors, particularly colorectal cancers, 5-FU has long been a mainstay chemotherapeutic antimetabolite drug. The response ratios of 5-FU monotherapy, combination therapy with irinotecan or oxaliplatin, and the newly developed combination therapy with bevacizumab and cetuximab are $15 \%$ (2), $40 \%$ (3), and $60-70 \%$ (4), respectively. However, the current therapy causes severe side-effects because it is not tumor-specific and it injures normal organs. Therefore, the development of cancer-specific therapies is urgently required.

The major mechanism of the cytotoxicity of 5-FU is the inhibition of nucleotide synthesis. This drug rapidly enters tumor cells, and one of the principal intracellular derivatives of 5-FU, fluorodeoxyuridine monophosphate (FdUMP), forms a covalent complex with thymidylate synthetase (TS), thereby inhibiting the catalytic activity of TS (5), leading to depletion of the intracellular pools of deoxythymidine mono- and tri-phosphate (dTMP and dTTP) and an increase in the relative levels of the normal precursor dUMP and its anabolic derivative dUTP (6). In addition to the nucleotide pool perturbations, UTP and FdUTP incorporate into DNA, resulting in the induction of stalled replication forks and S-phase arrest in cells treated with 5-FU (6,7). FdUTP and dUTP misincorporation 
is potentially mutagenic and miscoding, but both can be excised through the action of base excision repair (BER) and mismatch repair (MMR). DNA strand breaks are generated as byproducts of the repair processes, and such DNA damage can induce apoptosis if left unrepaired (8-10).

In response to DNA damage, cells activate a complex signaling network that mediates cell cycle arrest to allow time for DNA repair or, when the damage is extensive, to trigger apoptosis. The DNA damage response is initiated by activation of the ataxia telangiectasia mutated (ATM) and ATM- and Rad-related (ATR) kinases and the DNA-dependent protein kinase catalytic subunit. These kinases recruit the repair machinery to sites of damaged DNA while halting cell cycle progression by activating the effector kinases checkpoint kinase (Chk) 1 and 2. The activation of checkpoints controlled by ATM/ATR-Chk1/Chk2 stalls cell cycle progression in G1, $\mathrm{S}$, or G2 phase (11). G1 arrest is mediated by p53 through p21CIP1/WAF1 upregulation and, if the DNA damage is extensive, triggers apoptosis (12). However, many cancer cells show a loss of function of p53 or its regulatory pathways; therefore, chemotherapy-induced DNA damage fails to arrest the cancer cells in G1 phase and promote apoptosis. These cells are completely dependent on the $\mathrm{S}$ and $\mathrm{G} 2 / \mathrm{M}$ checkpoints to arrest the cell cycle and facilitate DNA repair before entry into mitosis (M phase) after genotoxic exposure. The ATR/Chk1 kinases are involved in the intra-S and G2/M checkpoints (13), function in the regulation of cell cycle arrest following genotoxic stress, and prevent new replication origins from firing during $\mathrm{S}$ phase.

Checkpoint kinase 1 (Chk1) inhibition induces the premature entry of cells with DNA damage into $M$ phase and leads to the promotion of abnormal mitotic spindles, altered chromosome segregation, abnormal cell division, the formation of multiple nuclei and apoptosis (14). This synergistic cytotoxicity of Chk1 inhibitors in combination with anticancer, DNA-damaging agents is especially effective against p53-deficient cancer cells compared with p53-proficient cells, including normal cells (15). These cancer-specific therapies have been considered examples of synthetic lethality, and many Chk1 inhibitors in combination with a variety of anticancer DNA-damaging agents are at various stages of preclinical and clinical development (16). In colorectal cancer, ATR, one of the regulators of Chk1 activation, is activated by DNA-damaging agents, and inhibition of ATR selectively sensitizes p53-deficient cells to cisplatin (17). It has also been reported that selective Chk1 inhibitors abrogate cell cycle checkpoints and potentiate the cytotoxicity of topoisomerase inhibitors in p53-deficient, but not in p53-proficient, colon cancer cells (18). 5-FU is the most frequently used chemotherapeutic agent for colorectal cancer. It has been reported that 5-FU activates Chk1 and that Chk1 downregulation abrogates S-phase arrest (19). Judging from these results, it is expected that the synergistic antitumor effects of Chk1 inhibition with 5-FU are more effective in p53-deficient cells than in p53-proficient colorectal cancer cells. However, these p53 status-dependent, synergistic antitumor effects of 5-FU and Chk inhibition in colorectal cancer are still unclear. Moreover, thus far, no therapy has reached the bedside, even though a highly selective Chk1 inhibitor would theoretically synergize with chemotherapy, suggesting the limitation of these therapies.
In this study, we investigated the effect of 5-FU treatment in p53-proficient and-deficient GI-tract cancer cells to develop tumor-specific anticancer therapy. In addition, we hypothesized that Chk1 inhibition might be effective in sensitizing 5-FU-resistant (5FUR) cancer cells to 5-FU because Chk1 activation is reported to be related to the resistance to chemotherapy (20). Therefore, we also investigated the synergistic cytotoxic potential of Chk1 inhibition on 5-FU treatment in p53-deficient colon cancer cells with/without 5-FU resistance. We observed that 5-FU specifically induced S-phase arrest in p53-deficient cancer cells, that 5-FU induced Chk1 activation and that Chk1 inhibition produced a synergistic effect on 5-FU cytotoxicity. We also found that in p53-deficient, 5FUR cancer, 5-FU did not induce S-phase arrest or Chk1 activation, although 5-FU induced significant DNA damage, and Chk1 inhibition did not sensitize the cells to 5-FU cytotoxicity.

\section{Materials and methods}

Cell lines and culture conditions. LS-174T and MKN45, which are wild-type p53 human colorectal and gastric cancer cell lines, respectively, and HT29, WiDr, and KATO III, which are p53-mutant human colorectal and gastric cancer cell lines, were obtained from the American Type Culture Collection (Rockville, MD, USA) and Riken Cell Bank (Ibaraki, Japan).

To prepare the 5FUR cancer cell line, HT29 was exposed to increasing doses of 5-FU, up to the clinically relevant plasma concentration of $2 \mu \mathrm{g} / \mathrm{ml}$. The surviving resistant cells were named 5FUR cells. All cells were cultured in RPMI-1640 medium containing $10 \%$ fetal bovine serum and $1 \%$ antibiotics and antimycotics at $37^{\circ} \mathrm{C}$ in a humidified atmosphere of $5 \% \mathrm{CO}_{2}$.

Drugs and antibodies. 5-FU was purchased from Sigma-Aldrich (St. Louis, MO, USA). SB218078, a Chk1 inhibitor, was obtained from Calbiochem (San Diego, CA, USA). The antibodies used for western blotting were as follows: rabbit polyclonal antibodies to phospho-ATR (Ser428), phospho-Chk1 (Ser296), and $\beta$-actin; rabbit monoclonal antibodies to phospho-ATM (Ser1981) and phospho-Chk1 (Ser345); and mouse monoclonal antibodies to Chk1 and TS (Cell Signaling Technology, Inc., Danvers, MA, USA).

Cell cycle analysis. Cells were seeded at $2.0 \times 10^{5}$ cells/well in 6-well plates and treated with or without 5-FU $(2 \mu \mathrm{g} / \mathrm{ml})$ and SB218078 $(1 \mu \mathrm{M})$ for the indicated time periods. The cell cycle profile was determined by the propidium iodide staining of nuclei isolated using the BD Cycletest Plus kit (BD Biosciences, San Jose, CA, USA) according to the supplier's directions. Fluorescence was quantitated using a FACSCanto ${ }^{\mathrm{TM}}$ flow cytometer with FACSDiva 6.1.3 software (BD Biosciences).

Cell survival assay. The rates of drug resistance were assessed using the WST assay with the Cell Count Reagent SF (Nacalai Tesque, Kyoto, Japan) according to the manufacturer's protocol. Briefly, 8,000 cells of each cell line were plated in each well of a 96-well plate in $100 \mu \mathrm{l}$ medium with or without 5-FU $(2 \mu \mathrm{g} / \mathrm{ml})$ for $48 \mathrm{~h}$. After treatment, $10 \mu \mathrm{l}$ WST reagent was added to each well, and the plate was incubated for $1 \mathrm{~h}$ 


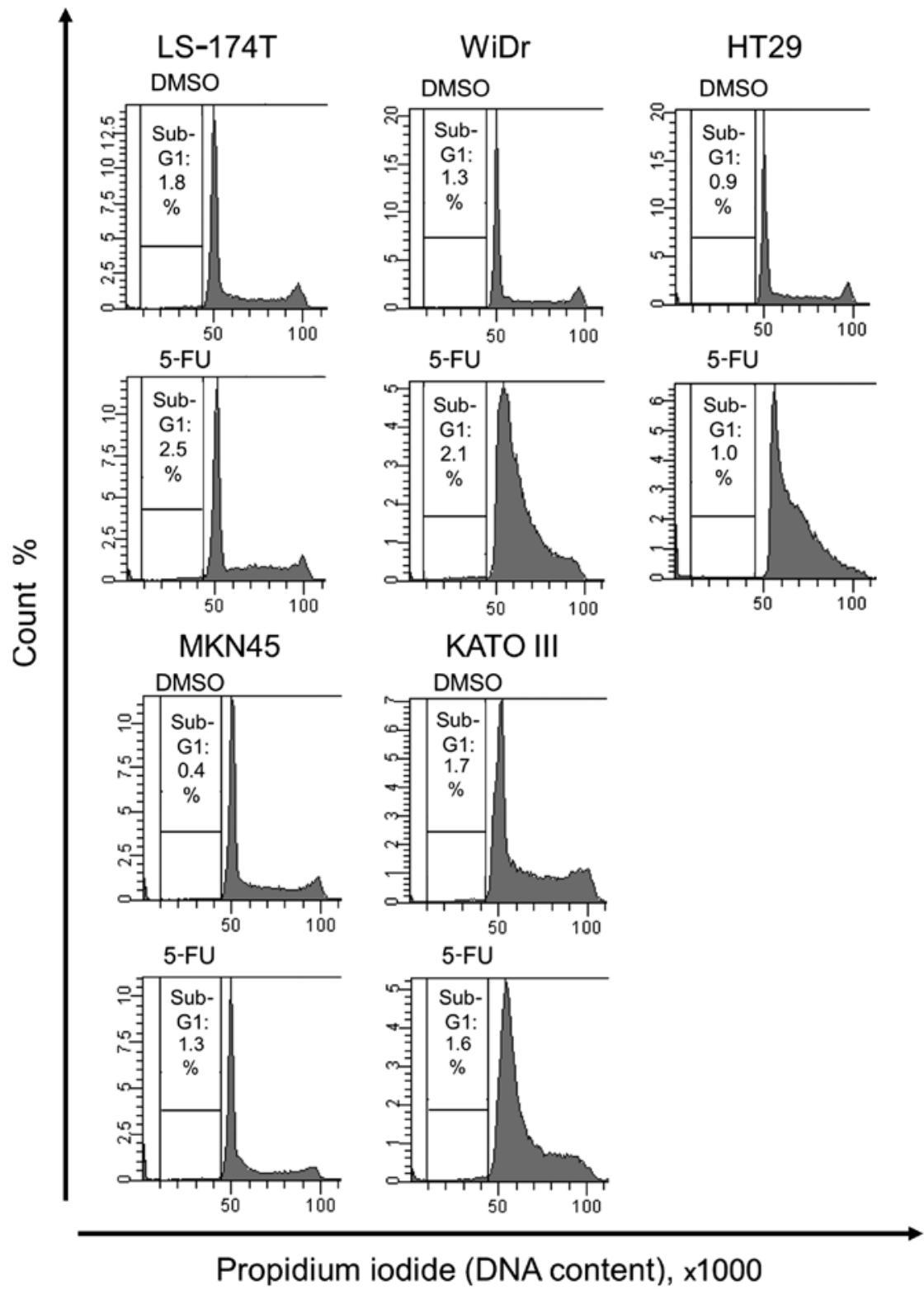

Figure 1. Cell cycle analysis of p53-wild and -mutant human gastrointestinal cancer cells treated with 5-FU. Analysis of the cell cycle of human colorectal and gastric cancer cell lines with wild-type or mutant p53 treated with or without $5-\mathrm{FU}(2 \mu \mathrm{g} / \mathrm{ml})$ for $48 \mathrm{~h}$. The cell cycle profile was determined by propidium iodide staining of isolated nuclei, and the fluorescence was quantitated. LS-174T and MKN45 are wild-type p53; WiDr, HT29, and KATO III are mutant p53.

at $37^{\circ} \mathrm{C}$. Colorimetric analysis was then performed at a wavelength of $450 \mathrm{~nm}$ using a standard microplate reader. Cell survival was calculated by dividing the surviving cell number estimated by WST in the presence of the drug by the number in the absence of the drug.

Alkaline comet assay. The alkaline comet assay was performed according to the method described by Singh et al (21), with slight modifications (22). After staining with $20 \mu \mathrm{g} / \mathrm{ml}$ ethidium bromide for $1 \mathrm{~min}$, we quantified the DNA damage of 100 randomly selected cells using the Comet Assay IV software (Perceptive Instruments Ltd., Suffolk, UK) on a computer attached to a fluorescence microscope (Olympus, Tokyo, Japan). We used the tail moment (the product of the relative intensity of the tail and the distance from the center of the nucleus to the center of gravity of the tail) to evaluate the degree of DNA damage.
Intracellular concentrations of 5-FU. The intracellular concentrations of 5-FU were measured by gas chromatography/mass spectrometry (GC/MS). Initially, cells were seeded at $1.2 \times 10^{6}$ cells $/ 10-\mathrm{cm}$ tissue culture dish and treated with 5-FU $(2 \mu \mathrm{g} / \mathrm{ml})$ for $48 \mathrm{~h}$. The GC/MS system consisted of a Trace GC gas chromatograph separation module and a Trace MS mass spectrometer (both from Thermo Fisher Scientific, Inc., Waltham, MA, USA). A DB-5 column (length, $30 \mathrm{~m}$; inside diameter, $0.32 \mathrm{~mm}$; film thickness, $0.25 \mu \mathrm{m}$; J\&W Scientific, Folsom, CA, USA) was used for the peak separation of 5-FU. The detector was used in SIM mode using the selected ion-monitoring procedure at $\mathrm{m} / \mathrm{z}=309$ for $5-\mathrm{FU}$ and at $\mathrm{m} / \mathrm{z}=311$ for $5-\mathrm{FU}-15 \mathrm{~N} 2$. An internal standard solution (including 5-FU-15N2) was added to each sample, and the solution was then extracted using ethyl acetate. The reaction product was extracted using a solution of mixed ethyl acetate and n-hexane, which was then evaporated to dryness under a 
A

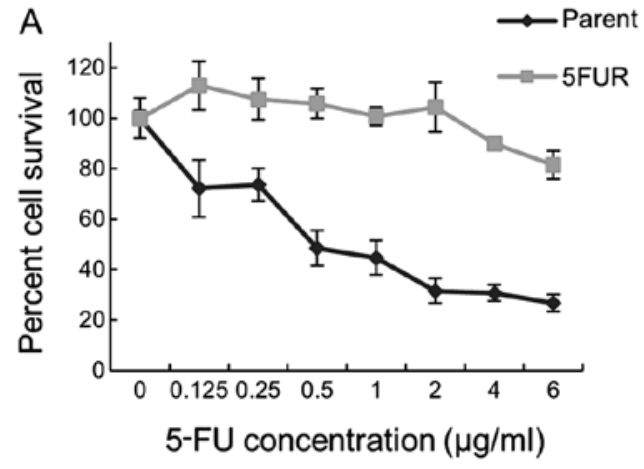

B

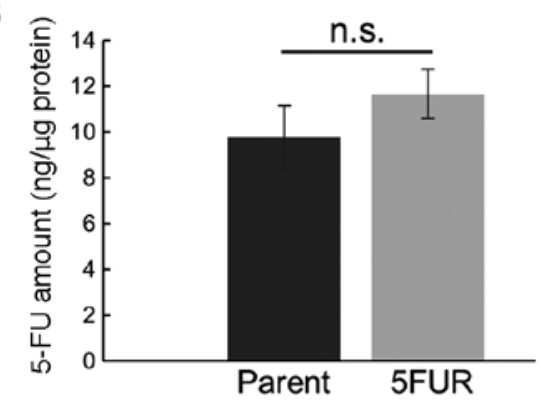

c
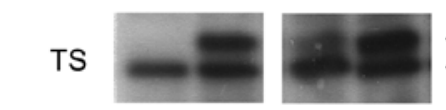

$\longleftarrow$ TS/FdUMP complex

$\beta$-actin

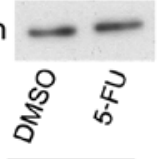

Parent

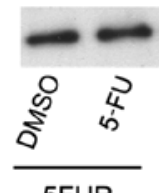

5FUR

D

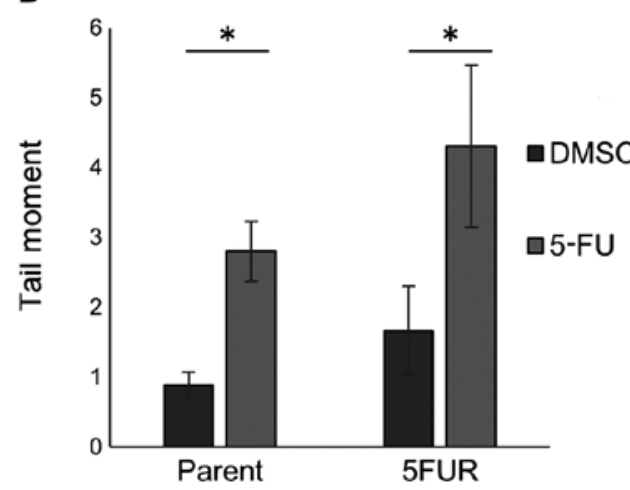

E

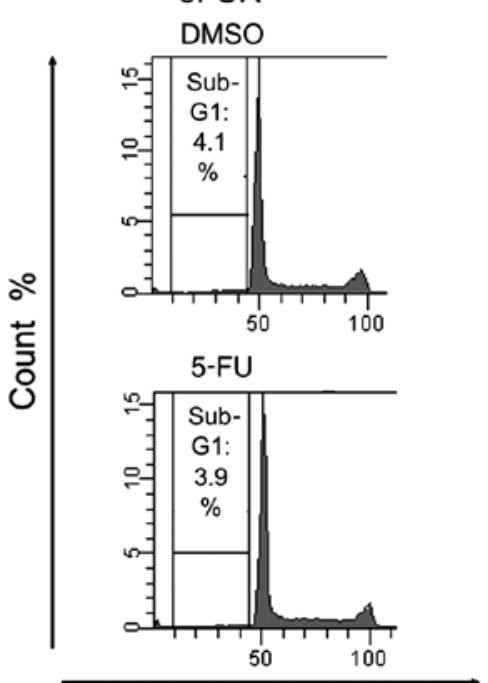

Propidium iodide (DNA content), $x 1000$

Figure 2. Characteristics of 5-FU-resistant (5FUR) cells. (A) The 5-FU sensitivity assay in parental and 5FUR cells. The WST assay was performed 48 h after treatment with 5-FU. (B) Intracellular concentrations of 5-FU, as measured by gas chromatography/mass spectrometry (GC/MS). Cells were treated with 5 -FU $(2 \mu \mathrm{g} / \mathrm{ml})$ for $48 \mathrm{~h}$. The intracellular concentrations of 5-FU were then measured by GC/MS. (C) Western blotting of thymidylate synthetase (TS) protein and the TS/FdUMP complex. The amounts of free TS were compared between parental cells treated with or without 5-FU for 48 h and 5FUR cells cultured with or without 5-FU for $48 \mathrm{~h}$. Complexes of fluorodeoxyuridine monophosphate (FdUMP) and TS were formed in the 5-FU-treated cells. (D) DNA damage assessed using a comet assay in cells treated with 5-FU. One hundred randomly selected cells stained with ethidium bromide were assayed by Comet Assay IV software. The product of the relative intensity of the tail and the distance from the center of the nucleus to the center of gravity of the tail was calculated to evaluate the degree of DNA damage. (E) Analysis of the cell cycle of parental and 5FUR cells treated with or without 5-FU for 48 h. The cell cycle profile was determined by propidium iodide staining of isolated nuclei, and the fluorescence was quantitated. ${ }^{*} \mathrm{P}<0.05$, relative to parental cells.

stream of nitrogen. The residue was dissolved in ethyl acetate, and a $1-\mu 1$ aliquot was injected into the GC/MS system.

Western blotting. For all western blotting, cells were seeded at $2.0 \times 10^{5}$ cells/well in 6-well plates and treated with or without 5-FU $(2 \mu \mathrm{g} / \mathrm{ml})$ and SB218078 $(1 \mu \mathrm{M})$ for $48 \mathrm{~h}$. The cells were lysed in lysis buffer [ $1 \%$ Nonidet P-40, $0.5 \%$ sodium deoxycholate, $0.1 \%$ sodium dodecyl sulfate, $1 \mathrm{x}$ protease inhibitor cocktail (Nacalai Tesque), and phosphate-buffered saline, $\mathrm{pH}$ 7.4]. Next, the lysates were cleared by centrifugation at $10,000 \mathrm{~g}$ at $4^{\circ} \mathrm{C}$ for $15 \mathrm{~min}$. The protein concentrations were determined using a bicinchoninic acid protein assay kit (Pierce Biotechnology, Inc., Rockford, IL, USA). Equal amounts $(10 \mu \mathrm{g})$ of the protein lysates were then electrophoretically separated on SDS-polyacrylamide gels and transferred onto a polyvinylidene fluoride membrane. For immunodetection, the above-mentioned primary antibodies were used.

\section{Results}

Cell cycle analysis of wild-type and mutant p53 human gastrointestinal cancer cells treated with 5-FU. At first, we investigated the effect of 5-FU treatment on the cell cycle of human colorectal and gastric cancer cell lines expressing wild-type p53 or mutant p53. In neither LS-174T nor MKN45 cells, which express wild-type p53, treated with 5-FU, no change was observed in $\mathrm{S}$ - or G2/M-phase compared with control cells. In contrast, 5-FU treatment induced S-phase arrest in HT29, WiDr, and KATO III cells, which express mutant p53 (Fig. 1).

Characteristics of 5FUR cells. To prepare 5FUR HT29 cells, dubbed 5FUR cells, HT29 cells were treated with increasing concentrations of 5-FU for 1 year. Fig. 2A shows the 5-FU sensitivity assay of the parental and 5FUR cells. 
The clinical 5-FU concentration in plasma is reported to be $<1.5 \mu \mathrm{g} / \mathrm{ml}$ (23); the survival of the 5FUR cells was significantly higher than that of the parental cells in the presence of $2 \mu \mathrm{g} / \mathrm{ml} 5-\mathrm{FU}(\mathrm{p}<0.05)$. There was no significant difference in the cellular 5-FU concentrations of the 5-FU-treated parental HT29 cells and the 5-FU-treated 5FUR cells (Fig. 2B). In certain parts of the patient population, an elevated TS level is linked to 5-FU resistance, so we compared the amount of free TS between parental cells and 5FUR cells treated with or without 5-FU for $48 \mathrm{~h}$. 5-FU treatment formed complexes of FdUMP and TS, and the residual 5-FU in the 5FUR cells formed a small amount of the complex. However, the amounts of free TS in the 5-FU-treated parental HT29 cells and the 5-FU-treated 5FUR cells were not different (Fig. 2C). We then used alkaline comet assays to analyze the levels of damaged DNA in the HT29 and 5FUR cells $48 \mathrm{~h}$ after treatment with $2 \mu \mathrm{g} / \mathrm{ml} 5$-FU. In the comet assays, both the parental and the 5FUR cells treated with 5-FU had longer tail moments than did the 5-FU-untreated parental and 5FUR cells (Fig. 2D). We also investigated the effect of 5-FU treatment on the cell cycle phase distribution of both cell types (Fig. 2E). The sub-G1 fraction was not detected in the parental HT29 or 5-FU-treated 5FUR cells, indicating that 5-FU did not induce apoptosis in either cell type. Additionally, 5-FU induced S-phase arrest in the parental HT29 cells, but in the 5FUR cells, 5-FU induced no change in the cell cycle compared with DMSO treatment, indicating that the acquisition of 5-FU resistance abrogated the 5-FU-induced S-phase arrest.

Acquisition of 5-FU resistance abrogates 5-FU-induced Chk1 phosphorylation. Chk1 regulates $\mathrm{S}$-phase arrest; therefore, we determined whether 5-FU activated the Chk1 pathway in both parental and 5FUR cells (Fig. 3). Chk1 activation is associated with phosphorylation at Ser345 (the site of Chk1 phosphorylation by ATR/ATM) and autophosphorylation at Ser296. The assessment of Chk1 activation (using a DNA-damaging agent or antimetabolite) and Chk1 inhibition (by a Chk1 inhibitor) is most likely best accomplished by monitoring the phosphorylation of Ser296 (15). In particular, SB218078 inhibits Chk1 autophosphorylation (Ser296) and increases the phosphorylation of ATM (Ser1981) and Chk1 (Ser345) (24). In this study, the phosphorylation of Chk1 and ATM (Ser1981) was not enhanced in 5FUR cells, whereas the phosphorylation of Chk1 and ATM was enhanced in parental cells treated with 5-FU. As a previous study reported (24), SB218078 significantly reduced the phosphorylation of Chk1 (Ser296), whereas the phosphorylation of Chk1 (Ser345) and ATM (Ser1981) was actually enhanced in parental and 5FUR cells with or without 5-FU treatment. In contrast, the phosphorylation of ATR (Ser428) was not enhanced by 5-FU treatment in parental or 5FUR cells.

Effect of the acquisition of 5-FU resistance on the synergistic effect of the Chk1 inhibitor SB218078 and 5-FU in HT29 cells. The synergistic effect of combined 5-FU and SB218078 treatment was evaluated by analyzing the cell cycle phase distribution and cell death rates. SB218078 showed no effect on cell viability in the parent cell line during monotherapy, but when in combination with 5-FU, SB218078 reduced the early S-phase arrest induced by 5-FU treatment and increased

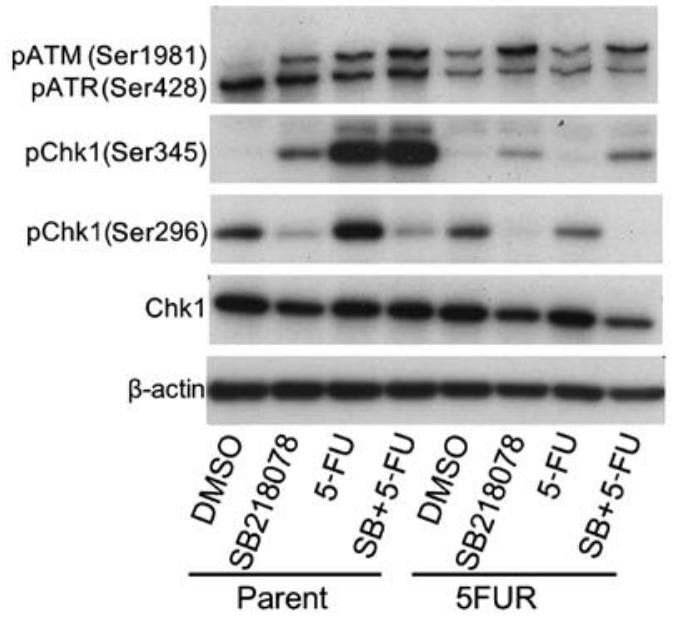

Figure 3. Western blotting to evaluate activation of ATM/ATR/Chk1 pathway in parental and 5-FU-resistant (5FUR) cells. Parental and 5FUR cells were exposed to 5-FU $(2 \mu \mathrm{g} / \mathrm{ml})$ and SB218078 $(1 \mu \mathrm{M})$ for $48 \mathrm{~h}$. Anti-phospho-ATR (Ser428) and anti-phospho-ATM (Ser1981) antibodies were then used to estimate the activation of ataxia telangiectasia mutated (ATM)- and Rad-related (ATR) and ATM kinases, respectively. Anti-phospho-Chk1 (Ser345) antibody was also used to estimate the phosphorylation of checkpoint kinase 1 (Chk1) by activated ATR or ATM. Another anti-phospho-Chk1 (Ser296) was used to estimate the activation of Chk1 by the autophosphorylation of Chk1 phosphorylated at Ser345. $\beta$-actin expression was determined to confirm equal protein loading.

the sub-G1 population, indicating induction of apoptotic cell death. In contrast, SB218078 exhibited no effect on cell death in 5FUR cell lines during monotherapy or when in combination with 5-FU (Fig. 4).

\section{Discussion}

In this study, we investigated the synergistic antitumor effect of a Chk1 inhibitor and 5-FU that was specific to p53-deficient cells, and we analyzed the potential of Chk1 inhibition to sensitize 5FUR cancer cells to 5-FU to develop a tumor-specific, effective therapy that overcame 5-FU resistance.

The enhanced excretion or degradation of 5-FU and the expression of TS are well-known mechanisms for 5-FU resistance. In this study, however, equal levels of intracellular 5-FU and free TS protein were detected in parental and 5FUR cells that were treated with $5-\mathrm{FU}$. Chk1 is overexpressed in a variety of human tumors, including breast, colon, liver, gastric, and nasopharyngeal carcinoma (25-31). Remarkably, Chk1 expression often positively correlates with tumor grade and disease recurrence and may contribute to therapy resistance $(29,30,32)$. The enhanced activation of Chk1 is also related to the resistance of cancer cells, including cancer stem cells from brain glioblastoma, prostate, and lung NSCLC, to chemotherapy or radiotherapy (33-38). Therefore, we hypothesized that 5FUR cells might possess more Chk1 activity than control cells and that Chk1 inhibition might exert a synergistic, cytotoxic effect and sensitize chemo-resistant cells to 5-FU.

DNA repair systems promote the faithful transmission of genomes in dividing cells by reversing extrinsic and intrinsic DNA damage and are required for cell survival during replication. Cancer cells are frequently found to be deficient in certain aspects of DNA repair. The impairment of DNA repair systems 


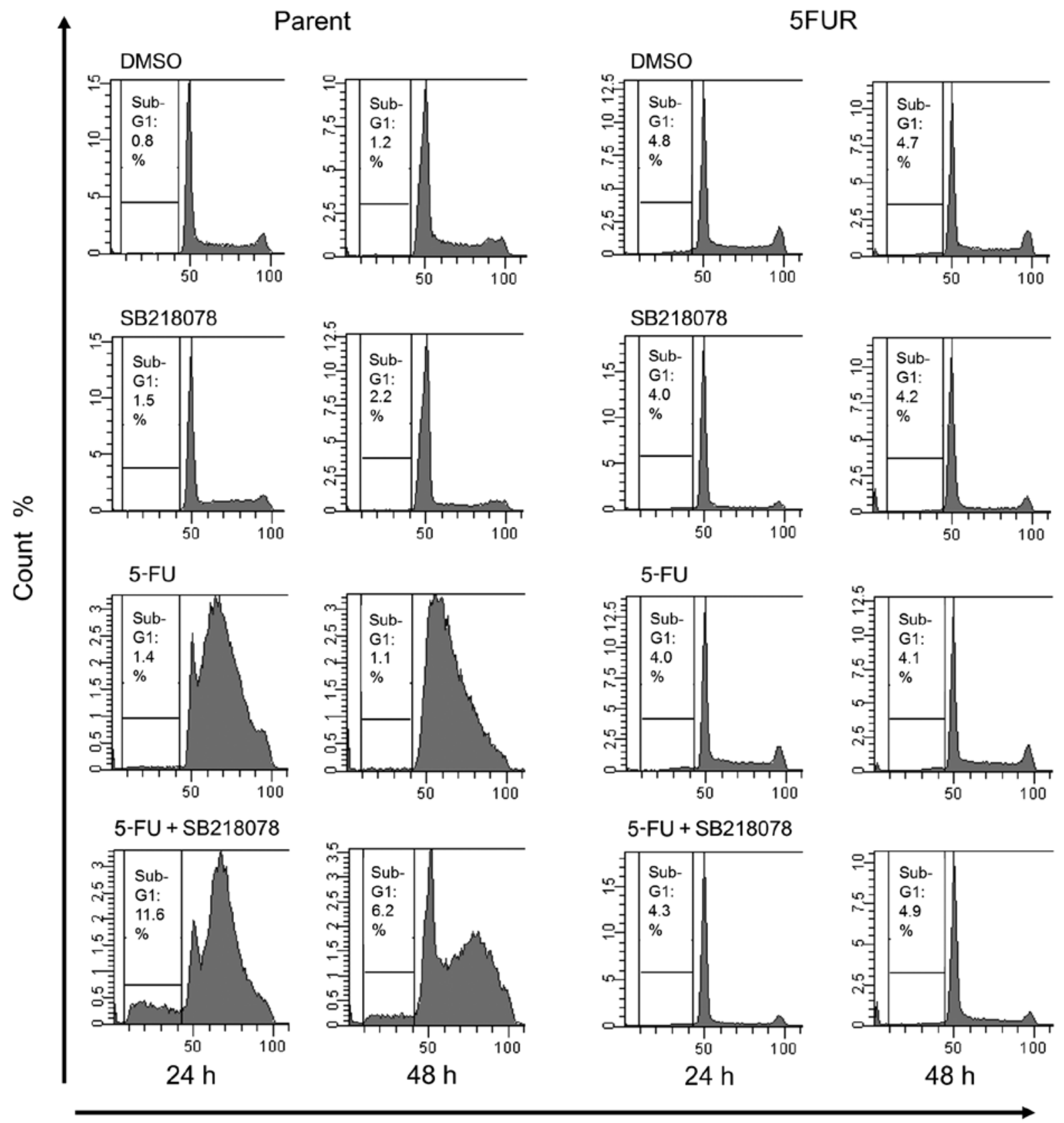

Propidium iodide (DNA content), $x 1000$

Figure 4. Analysis of cell cycle in presence of 5-FU and SB218078. After 24 and $48 \mathrm{~h}$ of drug treatment, DNA fragmentation was assessed by propidium iodide staining and flow cytometry analysis. The fluorescence of cells with sub-G1 DNA content, representing apoptotic cells, was quantitated.

contributes not only to the initial mechanism of carcinogenesis but also to its weakness, as these repair systems are required for the cancer cells to maintain their own survival (39).

Ovarian and breast cancer patients with BRCA mutations exhibit favorable responses to poly(ADP-ribose) polymerase (PARP) inhibitors compared with patients without BRCA mutations, as homologous recombination-deficient tumors can be effectively targeted by DNA double-strand break-inducing agents (40). This concept, that is, synthetic lethality, is attracting attention in the development of tumor-specific therapy. Synthetic lethal interactions are defined as two genetic alterations that cause cell death when they occur together, although neither mutation alone is lethal (41). The pharmacological inhibition of one gene product can be synthetically lethal when it occurs in combination with a pre-existing, cancer-related mutation, especially when the mutated cancer cells have become dependent on special pathways, leading to the ability to selectively target and kill the cancer cells while sparing the normal cells (42).

The development of anticancer regimens that take advantage of the molecular differences between normal and cancer cells is highly desirable. TP53 is one of the most frequently mutated genes in human cancers, so there is great interest in finding anticancer regimens that selectively target p53-deficient tumors (43). The cancer cells showing a loss of function of p53 or its regulatory pathways have a deficiency in the G1 checkpoint and are completely dependent on the $\mathrm{S}$ and G2/M checkpoints to arrest the cell cycle after genotoxic stress. 
Chk1 is critical for $\mathrm{S}$ and $\mathrm{G} 2 / \mathrm{M}$ arrest via downregulation of Cdc25A, cyclin A and CDK2 expression (44). Chk1 inhibition abolishes the $\mathrm{S}$ and $\mathrm{G} 2$ checkpoints induced by 5-FU, causes excessive accumulation of DNA damage, induces apoptosis, and ultimately selectively potentiates the efficacy of 5-FU in p53-deficient cells. In contrast, p53-dependent checkpoint(s) in p53-proficient cells allow DNA repair and thereby prevent sensitization to DNA damage (19).

In this study, we observed that 5-FU induced S-phase arrest only in p53-deficient cells and that 5-FU treatment induced Chk1 activation. We also found that Chk1 inhibition by SB218078 significantly increased the population of sub-G1 cells only in the presence of 5-FU. These results indicate that the development of tumor-specific anticancer therapy with 5-FU and Chk1 inhibitors for colorectal cancer, of which p53 is frequently mutated, is to be expected.

In the 5FUR cell line, 5-FU treatment did not induce Chk1 activation or S-phase arrest. Moreover, SB218078 in combination with 5-FU did not induce a sub-G1 population. These results revealed that in 5FUR, 5-FU-induced DNA damage induced neither Chk1 activation nor S-phase arrest, and 5FUR proliferated in the presence of 5-FU.

To understand the mechanisms underlying the abrogation of Chk1 activation in 5FUR cells, we examined the activation status of a major upstream regulator, Chk1, in these cells. More specifically, Chk1 is a traditional target of ATR in the DNA damage response. We observed that 5-FU treatment did not induce the activation of ATR in 5FUR cells or parental cells, although this treatment activated Chk1 in the parental cells. ATM is also required for Chk1 activation under certain circumstances (45-47). In this study, we found activation of ATM by 5-FU in parental cells, which demonstrates another example of ATM-Chk1 signaling mediating early S-phase arrest. In contrast, in 5FUR, neither ATR nor ATM was activated by DNA damage induced by $5-\mathrm{FU}$ treatment, leading to no induction of Chk1 activation, although there was no difference in the cellular 5-FU concentration or in the amount of free TS between the parental and 5FUR cells, indicating that 5-FU functioned in 5FUR cells as well as in parental cells. The precise mechanism of ATR, ATM, and Chk1 inactivation observed in 5FUR cells remains to be elucidated.

DNA-damaging agents combined with a Chk1 inhibitor cause tumor cells to undergo apoptosis in p53-deficient tumors (48). Mice with Chk1 disruption die during early development $(49,50)$, and the conditional deletion of Chk1 in proliferating mouse mammary epithelial cells induces apoptosis and developmental defects. In contrast to these studies, we did not observe increased apoptosis in 5FUR cells, in which 5-FU treatment induced DNA damage but did not induce Chk1 activation, cell cycle arrest, or an increased sub-G1 fraction. These results indicated that the 5FUR cells acquired apoptotic resistance. It has been reported that the overexpression of Bcl-xL suppresses Chk1 inhibitory lethality (51-53). The precise mechanisms of the anti-apoptotic potentials remain to be elucidated; however, in this study, we observed enhanced expression of Bcl-w and c-FLIP (data not shown) in 5FUR cells.

This study re-evaluated the key role of Chk1 in regulating the 5-FU-induced DNA damage checkpoint and the utility of Chk1 inhibition in tumor-specific anticancer therapy by enhancing the anticancer efficacy of 5-FU only in p53-deficient cells. However, 5-FU resistance in p53-deficient colorectal cancer cells abrogated 5-FU-induced Chk1 phosphorylation, S-phase arrest, and sensitization of p53-deficient cancer cells to 5 -FU by Chk1 inhibition. Therefore, Chk1 inhibition combined with 5-FU in p53-deficient cells does not appear to be a promising approach in the context of tolerance to 5-FU.

\section{References}

1. Marin JJ, Briz O, Monte MJ, Blazquez AG and Macias RI: Genetic variants in genes involved in mechanisms of chemoresistance to anticancer drugs. Curr Cancer Drug Targets 12: 402-438, 2012.

2. Johnston PG and Kaye S: Capecitabine: a novel agent for the treatment of solid tumors. Anticancer Drugs 12: 639-646, 2001.

3. Martin RC II, Scoggins CR, Tomalty D, et al: Irinotecan drug-eluting beads in the treatment of chemo-naive unresectable colorectal liver metastasis with concomitant systemic fluorouracil and oxaliplatin: results of pharmacokinetics and phase I trial. J Gastrointest Surg 16: 1531-1538, 2012.

4. Feng QY, Wei Y, Chen JW, et al: Anti-EGFR and anti-VEGF agents: important targeted therapies of colorectal liver metastases. World J Gastroenterol 20: 4263-4275, 2014.

5. Pinedo HM and Peters GF: Fluorouracil: biochemistry and pharmacology. J Clin Oncol 6: 1653-1664, 1988.

6. Longley DB, Harkin DP and Johnston PG: 5-fluorouracil: mechanisms of action and clinical strategies. Nat Rev Cancer 3: 330-338, 2003.

7. Sampath D, Rao VA and Plunkett W: Mechanisms of apoptosis induction by nucleoside analogs. Oncogene 22: 9063-9074, 2003.

8. Bartek J, Lukas C and Lukas J: Checking on DNA damage in S phase. Nat Rev Mol Cell Biol 5: 792-804, 2004.

9. Jackson SP and Bartek J: The DNA-damage response in human biology and disease. Nature 461: 1071-1078, 2009.

10. Aziz K, Nowsheen S, Pantelias G, Iliakis G, Gorgoulis VG and Georgakilas AG: Targeting DNA damage and repair: embracing the pharmacological era for successful cancer therapy. Pharmacol Ther 133: 334-350, 2012.

11. Zhou BB and Elledge SJ: The DNA damage response: putting checkpoints in perspective. Nature 408: 433-439, 2000.

12. Vogelstein B, Lane D and Levine AJ: Surfing the p53 network. Nature 408: 307-310, 2000.

13. Levesque AA and Eastman A: p53-based cancer therapies: is defective p53 the Achilles heel of the tumor? Carcinogenesis 28: 13-20, 2007.

14. Tao ZF and Lin NH: Chk1 inhibitors for novel cancer treatment. Anticancer Agents Med Chem 6: 377-388, 2006.

15. Ma CX, Janetka JW and Piwnica-Worms H: Death by releasing the breaks: CHK1 inhibitors as cancer therapeutics. Trends Mol Med 17: 88-96, 2011.

16. Maugeri-Saccà $M$, Bartucci $M$ and De Maria R: Checkpoint kinase 1 inhibitors for potentiating systemic anticancer therapy. Cancer Treat Rev 39: 525-533, 2013.

17. Sangster-Guity N, Conrad BH, Papadopoulos N and Bunz F: ATR mediates cisplatin resistance in a p53 genotype-specific manner. Oncogene 30: 2526-2533, 2011.

18. Chen Z, Xiao Z, Gu WZ, et al: Selective Chk1 inhibitors differentially sensitize p53-deficient cancer cells to cancer therapeutics. Int J Cancer 119: 2784-2794, 2006.

19. Xiao Z, Xue J, Sowin TJ, Rosenberg SH and Zhang H: A novel mechanism of checkpoint abrogation conferred by Chk1 downregulation. Oncogene 24: 1403-1411, 2005.

20. Zhang Y and Hunter T: Roles of Chk1 in cell biology and cancer therapy. Int J Cancer 134: 1013-1023, 2014.

21. Singh NP, McCoy MT, Tice RR and Schneider EL: A simple technique for quantitation of low levels of DNA damage in individual cells. Exp Cell Res 175: 184-191, 1988.

22. Lu Y, Morimoto K and Nakayama K: Health practices and leukocyte DNA damage in Japanese hard-metal workers. Prev Med 43: 140-144, 2006.

23. Yoshikawa R, Kusunoki M, Yanagi H, et al: Dual antitumor effects of 5-fluorouracil on the cell cycle in colorectal carcinoma cells: a novel target mechanism concept for pharmacokinetic modulating chemotherapy. Cancer Res 61: 1029-1037, 2001. 
24. Okita N, Minato S, Ohmi E, Tanuma S and Higami Y: DNA damage-induced CHK1 autophosphorylation at Ser296 is regulated by an intramolecular mechanism. FEBS Lett 586 3974-3979, 2012.

25. Sriuranpong V, Mutirangura A, Gillespie JW, et al: Global gene expression profile of nasopharyngeal carcinoma by laser capture microdissection and complementary DNA microarrays. Clin Cancer Res 10: 4944-4958, 2004.

26. Cho SH, Toouli CD, Fujii GH, Crain C and Parry D: Chk1 is essential for tumor cell viability following activation of the replication checkpoint. Cell Cycle 4: 131-139, 2005.

27. Madoz-Gúrpide J, Cañamero M, Sanchez L, Solano J, Alfonso P and Casal JI: A proteomics analysis of cell signaling alterations in colorectal cancer. Mol Cell Proteomics 6: 2150-2164, 2007.

28. Verlinden L, Vanden Bempt I, Eelen G, et al: The E2F-regulated gene Chk1 is highly expressed in triple-negative estrogen receptor/progesterone receptor/HER-2 breast carcinomas Cancer Res 67: 6574-6581, 2007.

29. Yao H, Yang Z and Li Y: Expression of checkpoint kinase 1 and polo-like kinase 1 and its clinicopathological significance in benign and malignant lesions of the stomach. Zhong Nan Da Xue Xue Bao Yi Xue Ban 35: 1080-1084, 2010 (In Chinese).

30. Hong J, Hu K, Yuan Y, et al: CHK1 targets spleen tyrosine kinase (L) for proteolysis in hepatocellular carcinoma. J Clin Invest 122: 2165-2175, 2012.

31. Xu J, Li Y, Wang F, et al: Suppressed miR-424 expression via upregulation of target gene Chk1 contributes to the progression of cervical cancer. Oncogene 32: 976-987, 2013.

32. Lundgren K, Holm K, Nordenskjöld B, Borg A and Landberg G: Gene products of chromosome 11q and their association with CCND1 gene amplification and tamoxifen resistance in premenopausal breast cancer. Breast Cancer Res 10: R81, 2008.

33. Perego P, Gatti L, Righetti SC, et al: Development of resistance to a trinuclear platinum complex in ovarian carcinoma cells. Int J Cancer 105: 617-624, 2003

34. Bao S, Wu Q, McLendon RE, et al: Glioma stem cells promote radioresistance by preferential activation of the DNA damage response. Nature 444: 756-760, 2006.

35. Cavelier C, Didier C, Prade N, et al: Constitutive activation of the DNA damage signaling pathway in acute myeloid leukemia with complex karyotype: potential importance for checkpoint targeting therapy. Cancer Res 69: 8652-8661, 2009.

36. Zhang YW, Brognard J, Coughlin C, et al: The F box protein Fbx6 regulates Chk1 stability and cellular sensitivity to replication stress. Mol Cell 35: 442-453, 2009.

37. Bartucci M, Svensson S, Romania P, et al: Therapeutic targeting of Chk1 in NSCLC stem cells during chemotherapy. Cell Death Differ 19: 768-778, 2012

38. Wang X, Ma Z, Xiao Z, et al: Chk1 knockdown confers radiosensitization in prostate cancer stem cells. Oncol Rep 28 2247-2254, 2012.
39. Brody LC: Treating cancer by targeting a weakness. N Engl J Med 353: 949-950, 2005.

40. Audeh MW, Carmichael J, Penson RT, et al: Oral poly (ADP-ribose) polymerase inhibitor olaparib in patients with BRCA1 or BRCA 2 mutations and recurrent ovarian cancer: a proof-of-concept trial. Lancet 376: 245-251, 2010.

41. Bouwman P and Jonkers J: The effects of deregulated DNA damage signalling on cancer chemotherapy response and resistance. Nat Rev Cancer 12: 587-598, 2012.

42. Dar AC, Das TK, Shokat KM and Cagan RL: Chemical genetic discovery of targets and anti-targets for cancer polypharmacology. Nature 486: 80-84, 2012.

43. Petitjean A, Mathe E, Kato S, et al: Impact of mutant p53 functional properties on TP53 mutation patterns and tumor phenotype: lessons from recent developments in the IARC TP53 database. Hum Mutat 28: 622-629, 2007.

44. Tu YS, Kang XL, Zhou JG, Lv XF, Tang YB and Guan YY: Involvement of Chk1-Cdc25A-cyclin A/CDK2 pathway in simvastatin induced S-phase cell cycle arrest and apoptosis in multiple myeloma cells. Eur J Pharmacol 670: 356-364, 2011.

45. Sahu RP, Batra S and Srivastava SK: Activation of ATM/Chk1 by curcumin causes cell cycle arrest and apoptosis in human pancreatic cancer cells. Br J Cancer 100: 1425-1433, 2009.

46. Jazayeri A,Falck J, Lukas C, et al: ATM- and cell cycle-dependent regulation of ATR in response to DNA double-strand breaks. Nat Cell Biol 8: 37-45, 2006.

47. Cuadrado M, Martinez-Pastor B, Murga M, et al: ATM regulates ATR chromatin loading in response to DNA double-strand breaks. J Exp Med 203: 297-303, 2006.

48. Eastman A: Cell cycle checkpoints and their impact on anticancer therapeutic strategies. J Cell Biochem 91: 223-231, 2004.

49. Liu Q, Guntuku S, Cui XS, et al: Chk1 is an essential kinase that is regulated by Atr and required for the G(2)/M DNA damage checkpoint. Genes Dev 14: 1448-1459, 2000.

50. Takai H, Naka K, Okada Y, et al: Chk2-deficient mice exhibit radioresistance and defective p53-mediated transcription. EMBO J 21: 5195-5205, 2002.

51. Bhonde MR, Hanski ML, Magrini R, et al: The broad-range cyclin-dependent kinase inhibitor UCN-01 induces apoptosis in colon carcinoma cells through transcriptional suppression of the Bcl-x(L) protein. Oncogene 24: 148-156, 2005.

52. Mitchell C, Park M, Eulitt P, Yang C, Yacoub A and Dent P. Poly(ADP-ribose) polymerase 1 modulates the lethality of CHK1 inhibitors in carcinoma cells. Mol Pharmacol 78: 909-917, 2010.

53. Tang Y, Dai Y, Grant S and Dent P: Enhancing CHK1 inhibitor lethality in glioblastoma. Cancer Biol Ther 13: 379-388, 2012. 